\title{
Research on Spectroscopy Modulation of a Distributed Feedback Laser Diode Based on the TDLAS Technique
}

\author{
Bin Li $\mathbb{D},{ }^{1,2}$ Liang Xue $\mathbb{D}^{2},{ }^{2}$ Nan Ji $\mathbb{D}^{2},{ }^{2}$ and Da Hui Wei $\mathbb{D}^{2}$ \\ ${ }^{1}$ College of Computer Science and Technology, Jilin University, Changchun 130012, China \\ ${ }^{2}$ Institute of Electrical \& Computer Engineering, Jilin Jianzhu University, Changchun 130012, China \\ Correspondence should be addressed to Bin Li; 870731919@qq.com
}

Received 11 September 2020; Revised 30 June 2021; Accepted 16 July 2021; Published 26 July 2021

Academic Editor: Mustapha Tlidi

Copyright (c) 2021 Bin Li et al. This is an open access article distributed under the Creative Commons Attribution License, which permits unrestricted use, distribution, and reproduction in any medium, provided the original work is properly cited.

Laser current and temperature control circuits have been developed for a distributed feedback laser diode, which is applied as the light source of a tuneable diode laser absorption spectroscopy system. The laser's temperature fluctuation can be limited within the range of -0.02 to $0.02^{\circ} \mathrm{C}$, and good operation stability was observed through 15 hours of monitoring on the emitting wavelength of the laser. Response time of temperature modulation was tested which is suitable for the tuning requirements of gas detection systems. Laser current can be injected within the range from 40 to $80 \mathrm{~mA}$. In addition, a linear power supply circuit has been developed to provide stable and low-noise power supply for the system. The physical principles of laser modulation theory are discussed before experiments. Experiments show that the output wavelength of the laser can be tuned accurately through changing the working current and temperature. The wavelength can be linearly controlled by temperature at $0.115 \mathrm{~nm} /{ }^{\circ} \mathrm{C}(I=70 \mathrm{~mA})$ and be controlled by current at $0.0140 \mathrm{~nm} / \mathrm{mA}\left(T=25^{\circ} \mathrm{C}\right)$. This is essential for the tuneable diode laser absorption spectroscopy systems. The proposed cost-effective circuits can replace commercial instruments to drive the laser to meet the requirements of methane detection experiments. It can also be applied to detect other gases by changing the light source lasers and parameters of the circuits.

\section{Introduction}

Infrared absorption spectroscopy is widely researched due to numerous advantages including fast response, nonintrusive nature, and sensitive species-specific detection capabilities [1-4], compared with traditional gas-sensing techniques such as catalyst combustion [5-7], electrochemical [8-10], and semiconductor [11-13]. Therefore, gas-sensing systems and sensors based on infrared absorption spectroscopy have been reported by many research groups in recent years, and many related techniques have been developed and improved to achieve high-sensitive sensing ability [14-16].

Tuneable diode laser absorption spectroscopy (TDLAS) technology is widely used in the field of trace gas detection, among many of the optical detection techniques [17-20]. In TDLAS systems, near-infrared (NIR) and mid-infrared (MIR) lasers are generally applied to be light sources. Distributed feedback (DFB) lasers are suitable for utilization in TDLAS systems and are especially used in the field of methane detection for the narrow line width and highly selective and reliable features [21-23]. In addition, the cost of DFB lasers is relatively much lower than that of midinfrared lasers and DFB lasers have the potential of remote multipoint probing by using optical fibre.

Tuneable laser diodes have three major parts: the excited region of active gain region and resonant cavity, wavelength tuning unit, and wavelength locking unit. First, wavelength can be changed by the resonant cavity. Then, frequency and phase of output wavelength can be modified and stabilized. The wavelength tuning unit is responsible for changing and selecting output wavelength. And the wavelength lock unit can stable the selected output wavelength [24]. Lasers can be catalogued according to their structures and processing technology. DFB lasers are tuneable laser diodes and are adopted in this paper. The output wavelength of DFB lasers is determined by the grating period $\Lambda$ and effective refractive 
index. Due to the reason that the effective refractive index is influenced by temperature, the output wavelength can be determined by temperature. In this way, the maximum tuning scope of a DFB laser is determined by the maximum scope of grating material's effective refractive index [25]. Generally, the current-wavelength tuning ratio is about $0.01 \mathrm{~nm} / \mathrm{mA}$. The temperature-wavelength tuning ratio is much bigger and is about $0.1 \mathrm{~nm} /{ }^{\circ} \mathrm{C}$.

DFB lasers are controlled by using laser driver devices that can set the working current and temperature. In this way, experiments based on TDLAS are commonly utilizing commercial instruments including laser current drivers and temperature controllers to satisfy the experimental requirements. Besides, a signal generator is also a type of essential instrument for a laser's output signal control. By utilizing the commercial instruments mentioned above, lasers can be controlled effectively to meet the requirements of gas-sensing experiments based on infrared absorption spectroscopy techniques $[26,27]$. However, there are still some drawbacks of using the commercial instruments. For instance, these systems are generally expensive and large. These drawbacks are practical barriers for industrialization of TDLAS-based gas-sensing technique, and these problems should be solved.

In this paper, a cost-effective DFB laser driver was developed and experiments were demonstrated. This laser driver is made of self-developed circuits. It has the capability of controlling laser temperature, driving current, and modulating essential signals based on the TDLAS technique. The modulated DFB laser can be applied as the radiation source of gas detection system. In order to provide stable and low-noise power supply, a linear power supply circuit has been developed to satisfy the system requirements. Spectroscopy measurement has been carried out to evaluate and optimize the performance of the DFB driving circuits and control algorithm. The applied gas-sensing theory is briefly discussed only to guide the audience and define units. Then, details of the key modules developed in this paper are demonstrated with individual tests. In addition, the modulation theory of lasers by current and temperature has been discussed in order to provide theoretical basis for circuits' design and experiments. Finally, the spectroscopy is measured by using the self-developed laser driver and the results are discussed in the end of this paper.

\section{Theory of Spectroscopy Modulation}

Modulation and implementation of a near-infrared DFB diode laser are generally easier and cheaper than those of a $3.31 \mu \mathrm{m}$ DFB diode laser, though $\mathrm{CH}_{4}$ molecules have larger absorption intensity at the wavelength of $3.31 \mu \mathrm{m}$, which is in the middle infrared domain. The laser diode adopted in this paper is a near-infrared distributed feedback laser diode, and its centre wavelength is shown in Figure 1. DFB lasers are mainly manufactured in the near-infrared domain and middle-infrared domain, and the output signal can be transmitted by using optical fibres. In this paper, the DFB laser's absorption band around $1.65 \mu \mathrm{m}$ is selected in $\mathrm{CH}_{4}$ detection. Figure 1(a) depicts the absorption spectrum of
$\mathrm{CH}_{4}$ molecules around $1.654 \mu \mathrm{m}$, and the intensity is at the level of $10^{-21} \mathrm{~cm}^{-1} /\left(\right.$ molecule $\left.\mathrm{cm}^{-2}\right)$. As can be seen from the comparison among Figures 1(a)-1(c), since the absorption intensities of $\mathrm{H}_{2} \mathrm{O}$ and $\mathrm{CO}_{2}$ are both 4 orders smaller than that of $\mathrm{CH}_{4}, \mathrm{H}_{2} \mathrm{O}$ and $\mathrm{CO}_{2}$ cause extremely slight effect on $\mathrm{CH}_{4}$ detection, and the detailed absorption band used in detection is shown in Figure 1(d) for a clearer illustration. Therefore, the centre wavelength of the DFB laser can be tuned across the absorption line of $\mathrm{CH}_{4}$ by changing its working current and temperature and the optical power loss caused by $\mathrm{H}_{2} \mathrm{O}, \mathrm{CO}_{2}$, and other gases can be ignored. Hence, detection selectivity of the $\mathrm{CH}_{4}$ TDLAS system can be guaranteed.

The schematic of this TDLAS-based methane detection system is shown in Figure 2. The proposed system is a typical TDLAS-based system, which consists of electrical circuit boards, DFB laser, fibre optic beam splitter (FOBS), optical attenuator (OA), InGaAs detectors, and input/output interfaces. The DFB laser, which is 14-pin butterfly-packaged, has a central wavelength around $1.654 \mu \mathrm{m}$. A digital signal processor (DSP) chip (TMS320F28335) is applied as the main controller of the developed DFB driver. It controls the laser's temperature, driving current, and modulating signals by sending signals to corresponding circuits such as TEC controlling circuit and DAC circuit. Meanwhile, it also processes signals that are related to gas concentration. Two InGaAs detectors are applied to convert optical signal to electrical signal. The yellow lines in Figure 2 represent optical fibres. The laser driving functions including temperature control, current control, and signal modulation are included together in this figure and are proposed in this paper.

The fundamental of absorption spectroscopy is based on the attenuation of spectral intensity $\left(\mathrm{W} / \mathrm{m}^{2}\right)$ occurring when radiation interacts with species molecules and is governed by the Beer-Lambert law, which relates the transmitted intensity $I(v)$ to the incident intensity $I_{0}(v)$ as follows:

$$
\tau(v, t)=\frac{I(v)}{I_{0}(v)}=\exp [-\alpha(v, t) L C],
$$

where $\alpha(v, t)\left(\mathrm{cm}^{-1}\right)$ is the absorption coefficient at frequency $v\left(\mathrm{~cm}^{-1}\right), C$ is the mole fraction of the absorbing specie, and $L$ is the optical path length. In application of TDLAS with WMS, the DFB laser is tuned with a lowfrequency repetitive ramp waveform with the period of $T_{\text {ramp. }}$. This has the effect of repetitively ramping the laser intensity and the laser wavelength across the absorption feature. In addition, a high-frequency sine wave signal with an angular frequency of $\omega_{\text {sin }}$ is superimposed on the repetitive injection current ramp to generate an additional high-frequency modulation [20]. The expressions of the ramp signal and the sine signal are represented in equations (2) and (3), respectively.

$$
\begin{gathered}
u_{\text {ramp }}(t)=a+\frac{a}{T_{\text {ramp }}}\left(t-T_{\text {ramp }}\right), \\
u_{\text {sin }}(t)=b \sin \left(\omega_{\sin } t\right), \\
I(t)=I_{0}\left[1+m u_{\text {ramp }}(t)+m u_{\text {sin }}(t)\right] .
\end{gathered}
$$




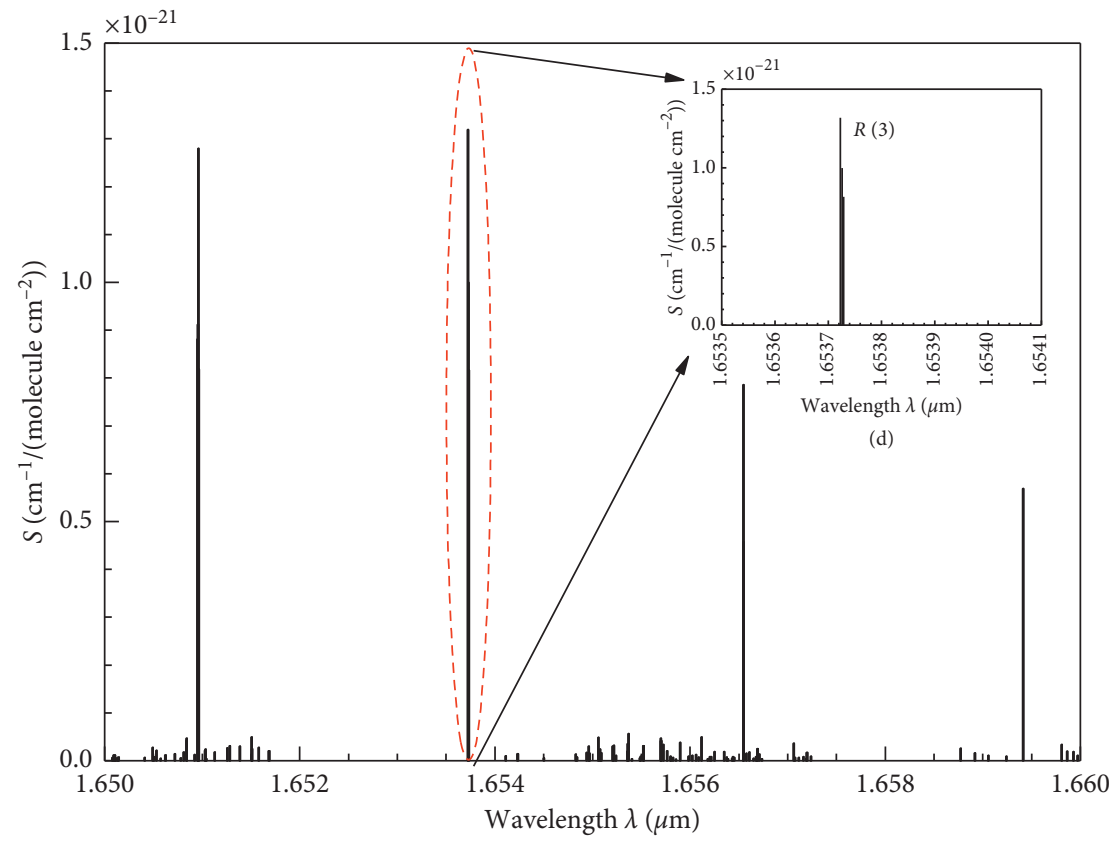

(a)

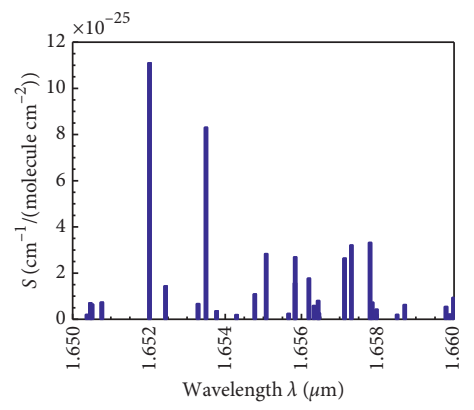

(b)

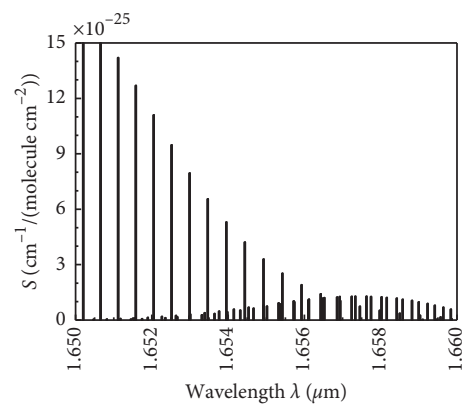

(c)

Figure 1: Absorption line selection and comparison among $\mathrm{CH}_{4}(\mathrm{a}, \mathrm{d}), \mathrm{H}_{2} \mathrm{O}$ (b), and $\mathrm{CO}_{2}$ (c).

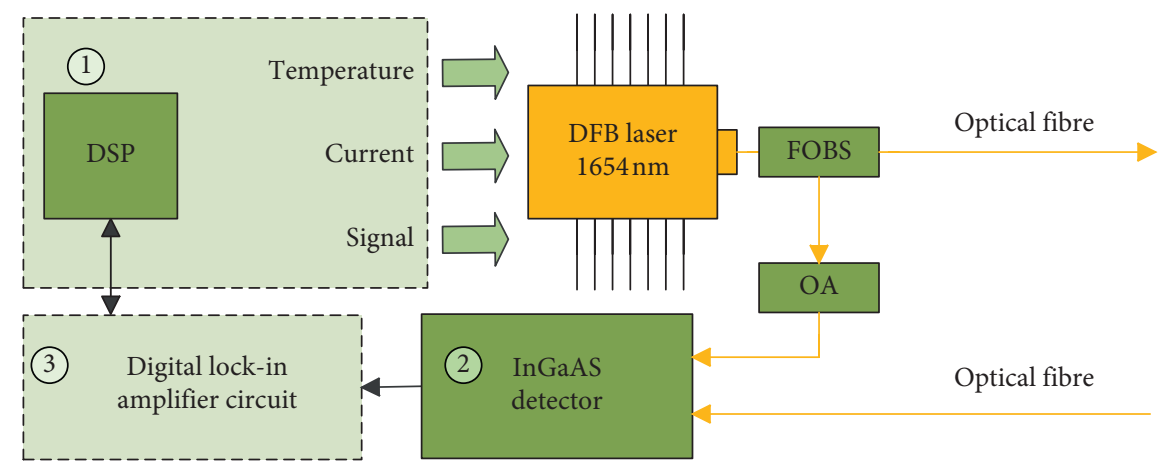

FIgUre 2: Schematic of the TDLAS-based gas detection system using the DFB laser at $1654 \mathrm{~nm}$.

\section{Materials and Methods}

3.1. Laser Temperature Controller. Generally, there are four ways to change the frequency of tuneable lasers as shown in Table 1. First, the plasma effect of carrier is caused by injection current. Second, electric field effect is known as the quantum confined Stark effect (QCSE). Third, temperature effect is widely adopted in TDLAS systems. Finally, mechanical effect is commonly adopted for the vertical cavity surface-emitting laser (VCSEL) [28-31]. It can be seen in Table 1 that the temperature effect is based on changing the effective refractive index in order to change the frequency. It is effective to modulate the DFB laser, and it is fordable to develop through the thermo electric cooler (TEC) control circuits for temperature controlling.

The resonance condition of the laser diode can be expressed as shown in

$$
m \lambda=2 n_{\mathrm{eff}} L .
$$

Here, $\lambda$ is the laser wavelength, $m$ is the resonant modes factor, $n_{\text {eff }}$ is the effective refractive index of active medium, and $L$ is the effective length of the active dielectric resonator. Therefore, the relative variation of wavelength modulation can be shown as

$$
\frac{\Delta \lambda}{\lambda}=\frac{\Delta n_{\text {eff }}}{n_{\text {eff }}}+\frac{\Delta L}{L}-\frac{\Delta m}{m} .
$$

Here, wavelength modulation can be achieved by using current modulation or temperature modulation in order to change the parameters including $\Delta n_{\text {eff }}, \Delta L$, and $\Delta m$. Current modulation is based on changing the injection current on electrodes in order to change the density of free carriers. And wavelength modulation can be performed by the variation of effective refractive index caused by plasma effect. Temperature modulation is similar to current modulation, and the effective refractive index can be changed by temperature variation in order to change the wavelength. In this paper, 
TABLE 1: Comparison of characteristics among four wavelength modulation methods.

\begin{tabular}{lcccc}
\hline Parameter & Plasma effect & Electric field effect & Temperature effect & Mechanical effect \\
\hline$\Delta n$ & -0.04 & -0.01 & -0.01 & - \\
$\Delta \lambda$ & $-8 \mathrm{~nm}$ & $-1 \mathrm{~nm}$ & $+5 \mathrm{~nm}$ & Depends on gain spectrum \\
Response time & $\sim \mathrm{ns}$ & $\sim \mathrm{ps}$ & $\sim \mathrm{ms}$ & $\sim$ ms \\
Heat value & High & Very low & Very high & None \\
Technical difficulty & Medium & Medium & Simple & Complex \\
\hline
\end{tabular}

both methods are applied to modulate wavelength by using the developed circuits.

Temperature control for the DFB laser is crucial for gas detection due to the fact that the laser's central wavelength and optical power are determined by the laser's operation temperature and working current. Thus, a temperature controller circuit was developed to meet the requirements of control features including stable, accurate, and quick response. Based on the Peltier effect, the laser's temperature can be set through the TEC module, which is integrated in the DFB laser. The DFB laser's temperature can be obtained by an inside-packaged thermistor with negative temperature coefficient (NTC). A high-efficiency and reliable monolithic TEC controller, ADN8831, is adopted to drive the TEC. It sets the temperature of the laser diode in a programmable way and maintains the temperature stable by using an analog PID control loop. The developed circuit is shown in Figure 3. It is a four-layer PCB, and the internal layers are prepared for power supply and GND. The parts on the board were soldered manually.

The temperature compensation network is shown in Figure 3. The values of $R_{\mathrm{LOW}}, R_{\mathrm{MID}}$, and $R_{\mathrm{HIGH}}$ can be determined, and the voltage $V_{\mathrm{SET}}$, which determines the laser temperature, can be calculated using

$$
\begin{aligned}
V_{\mathrm{SET}} & =\left(\frac{R_{3}}{R_{\mathrm{TH}}+R_{2}}-\frac{R_{3}}{R_{1}}+1\right) \times \frac{V_{\mathrm{REF}}}{2}, \\
R_{1} & =R_{\mathrm{MID}}+\frac{R_{\mathrm{MID}}\left(R_{\mathrm{LOW}}+R_{\mathrm{HIGH}}\right)-2 R_{\mathrm{HIGH}} R_{\mathrm{LOW}}}{R_{\mathrm{HIGH}}+R_{\mathrm{LOW}}-2 R_{\mathrm{MID}}}, \\
R_{2} & =R_{1}-R_{\mathrm{MID}}, \\
R_{3} & =\frac{R_{1}\left(R_{1}+R_{\mathrm{LOW}}-R_{\mathrm{MID}}\right)}{R_{\mathrm{LOW}}-R_{\mathrm{MID}}} .
\end{aligned}
$$

where $R_{\mathrm{LOW}}, R_{\mathrm{MID}}$, and $R_{\mathrm{HIGH}}$ depend on the temperature range, $R_{\mathrm{TH}}$ is the resistance of the thermistor, $V_{\mathrm{REF}}$ is the reference voltage, $R_{1}$ and $R_{2}$ are calculated using equations (8) and (9), and $R_{3}$ represents the compensation resistor. The schematic of temperature setting and compensating network is shown in Figure 3, where the resistors and capacitors, CD, $\mathrm{RD}, \mathrm{RI}, \mathrm{CI}, \mathrm{RP}$, and CF, set the analog PID loop. In order to evaluate the performance of temperature control, an evaluation board was developed as shown in Figure 3 .

Experiments were carried out to investigate the temperature control performances. Figure 4 shows the stability of the temperature control module. During a period of 15 hours, the operation temperature of the DFB laser is set as $24.55^{\circ} \mathrm{C}$. The practical temperature is sampled by an analogto-digital circuit, and a random period of 100 minutes is selected to see the details. It can be seen that the temperature is fluctuated within the range of $\pm 0.02^{\circ} \mathrm{C}$, which is small enough to avoid laser wavelength fluctuation.

A response time experiment was carried out to evaluate the performance of the laser temperature control circuit, and the result is shown in Figure 5. The response time test was performed by increasing and decreasing the temperature with the step of $1^{\circ} \mathrm{C}$. It can be seen in the figure that the response time is less than 8 seconds and the overheating and overcooling range is within $3^{\circ} \mathrm{C}$ without repeating thermal vibration. According to Table 1, laser frequency can be controlled by temperature and the response time is at the millisecond level. However, it requires more time to stabilize the working temperature of the laser after changing its value. It can be acceptable to have the temperature stabilized in the period of a few seconds. Therefore, experiment results suggest good performance of the developed laser temperature controller.

3.2. Linear Power Supply. In order to guarantee the operation stability and protect the DFB laser, a stable and lowripple linear power supply was developed and integrated, whose photo is shown in Figure 6. This power supply module possesses features including voltage regulation and low ripple, and these features are indispensable, especially for the temperature control module. The measured current variation is less than $\pm 2 \mathrm{mV}$. Electrostatic discharge (ESD) protection and surge protection are also put into use in this power supply system to protect the DFB laser. There are two circuit boards in this part. The parts on the boards were soldered manually. The output voltage has been set as $5 \mathrm{~V}$ and $\pm 12 \mathrm{~V}$ as shown in the photo.

3.3. Laser Current Controller. The DFB laser applied in this detection system is provided by the Institute of Semiconductors, Chinese Academy of Sciences (ISCAS). Its centre emitting wavelength is around $1654 \mathrm{~nm}$, and the laser photo is shown in Figure 7. It is 14-pin butterfly-packaged and has been designed for high-sensitivity optical sensor development. A negative temperature coefficient thermistor and a thermoelectric cooler are packaged inside for temperature monitoring and controlling. The threshold current is $17.23 \mathrm{~mA}$, and the optical output power is $5.34 \mathrm{~mW}$, while the injection current is $60 \mathrm{~mA}$ at $25^{\circ} \mathrm{C}$. The driving current is set within the range of $40-80 \mathrm{~mA}$, and the current varying step is $0.02 \mathrm{~mA}$. 


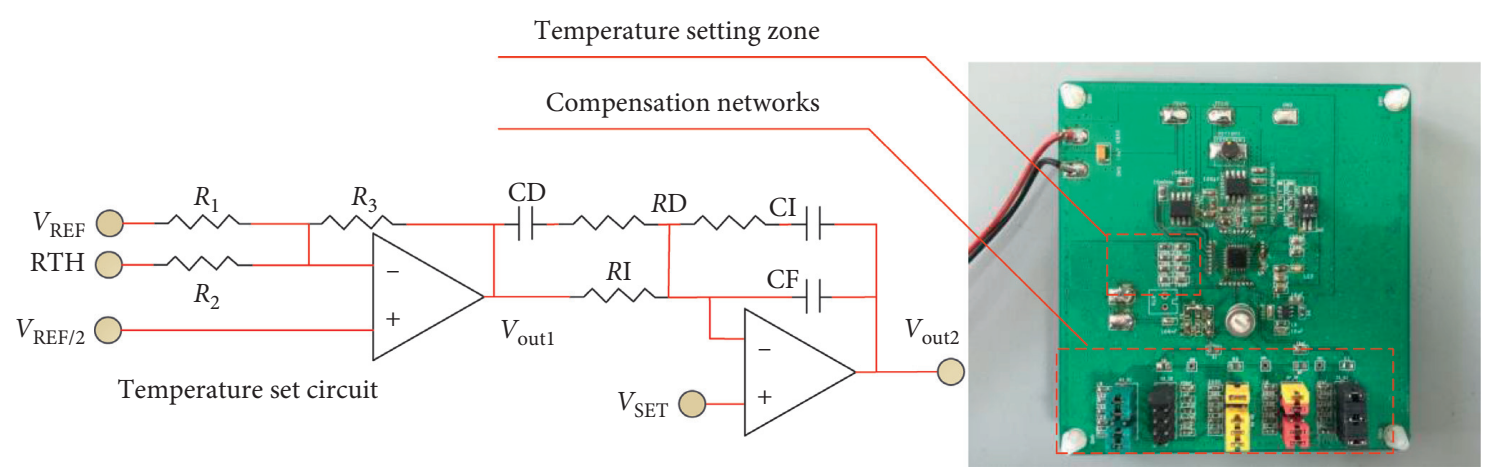

FIgURE 3: Laser temperature control and modulation board using ADN8831.

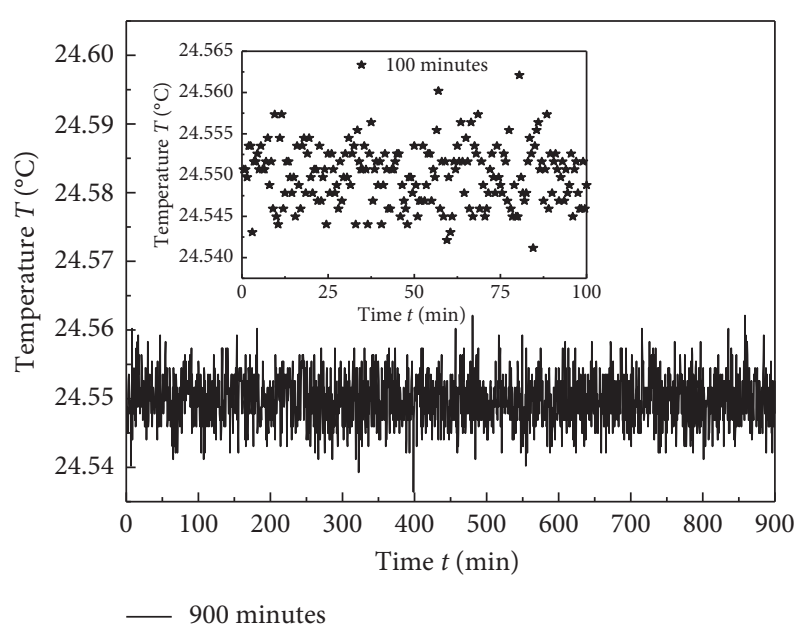

Figure 4: Temperature stability test during 15 hours.

The integrated board has been developed contenting functions including laser current control, laser temperature control, laser signal modulation, and communication functions as shown in Figure 7. The main controller of this circuit is a DSP chip, which is responsible for processing the calculations of laser current control, laser temperature control, and other functions. This 4-layer circuit board was also selfdeveloped by using the software Cadence. The output power of the laser is detected by using an optical power meter as shown in Figure 7. It can be seen that the optical power is $10.4 \mathrm{~mW}$, which is the maximum optical power of this DFB laser diode. Further experiments were carried out to evaluate the developed circuits by using this board.

\section{Spectroscopy Experiments}

4.1. Laser Temperature Robust Test. In order to evaluate the laser temperature control performance of the developed circuit, a laser temperature robust test has been carried out as shown in Figure 8. In this test, the laser is constantly heated by using a heat gun to simulate its working condition. This test repeated twice from the beginning to the end in a period of 200 seconds, and the laser temperature control circuit was switched. On the one hand, as shown in Figure 8(a), the black line demonstrates the laser

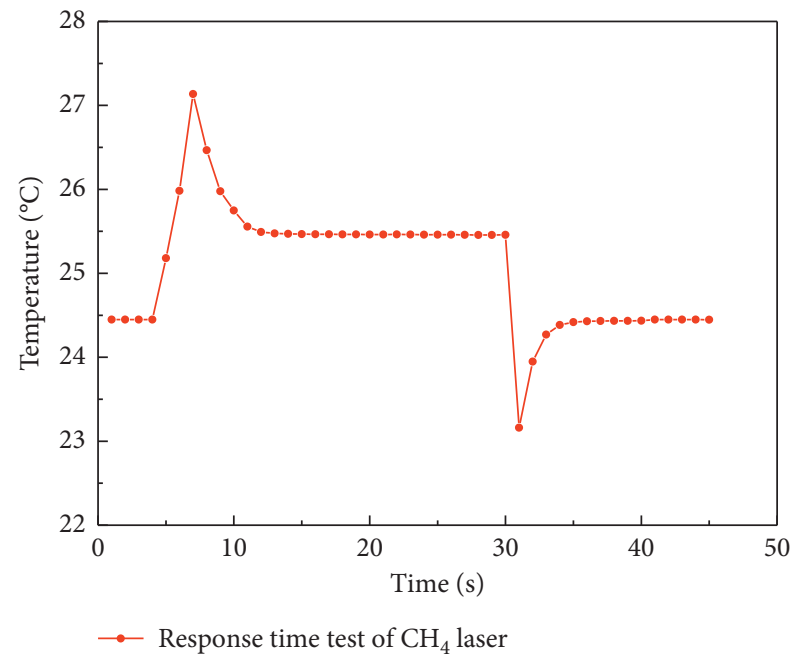

Figure 5: Response time test by using step as $1^{\circ} \mathrm{C}$.

temperature without the control circuit. It can be seen that the laser temperature maintains at about $27^{\circ} \mathrm{C}$, which is the room temperature, and it increases sharply continually when it was heated at the 80th second. On the other hand, the test was repeated again with the temperature control circuit as shown of the red line in Figure 8(a). It can be seen that the laser temperature maintains at the same level. The enlarged curve from 80 to 200 second is shown in Figure 8(b). It can be seen that the laser temperature started to fluctuate when it was heated, but the temperature has been controlled to its target by the analog PID circuit. In this way, the output centre wavelength of the laser can be maintained with the laser temperature control circuit.

4.2. Spectroscopy Test with Varying Laser Temperature. The centre wavelength of the laser is determined by the working current and temperature according to the previous descriptions. The relation between the centre wavelength and the temperature has been tested by using the developed circuit, and the results are shown in Figure 9. The laser current was set by the DSP circuit via DAC and VCCS circuits. In this figure, it can be seen that the laser wavelength increases linearly corresponding to the increasing 


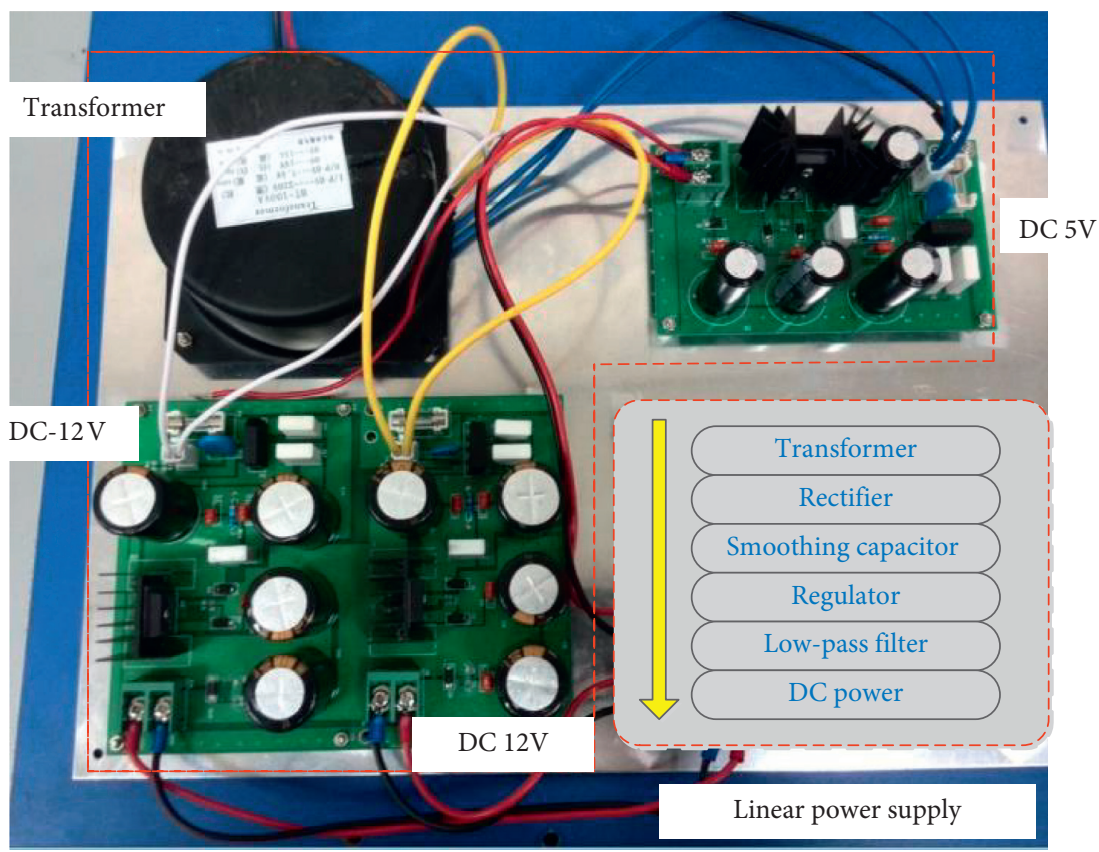

FIGURE 6: Photo of the self-developed linear power supply.

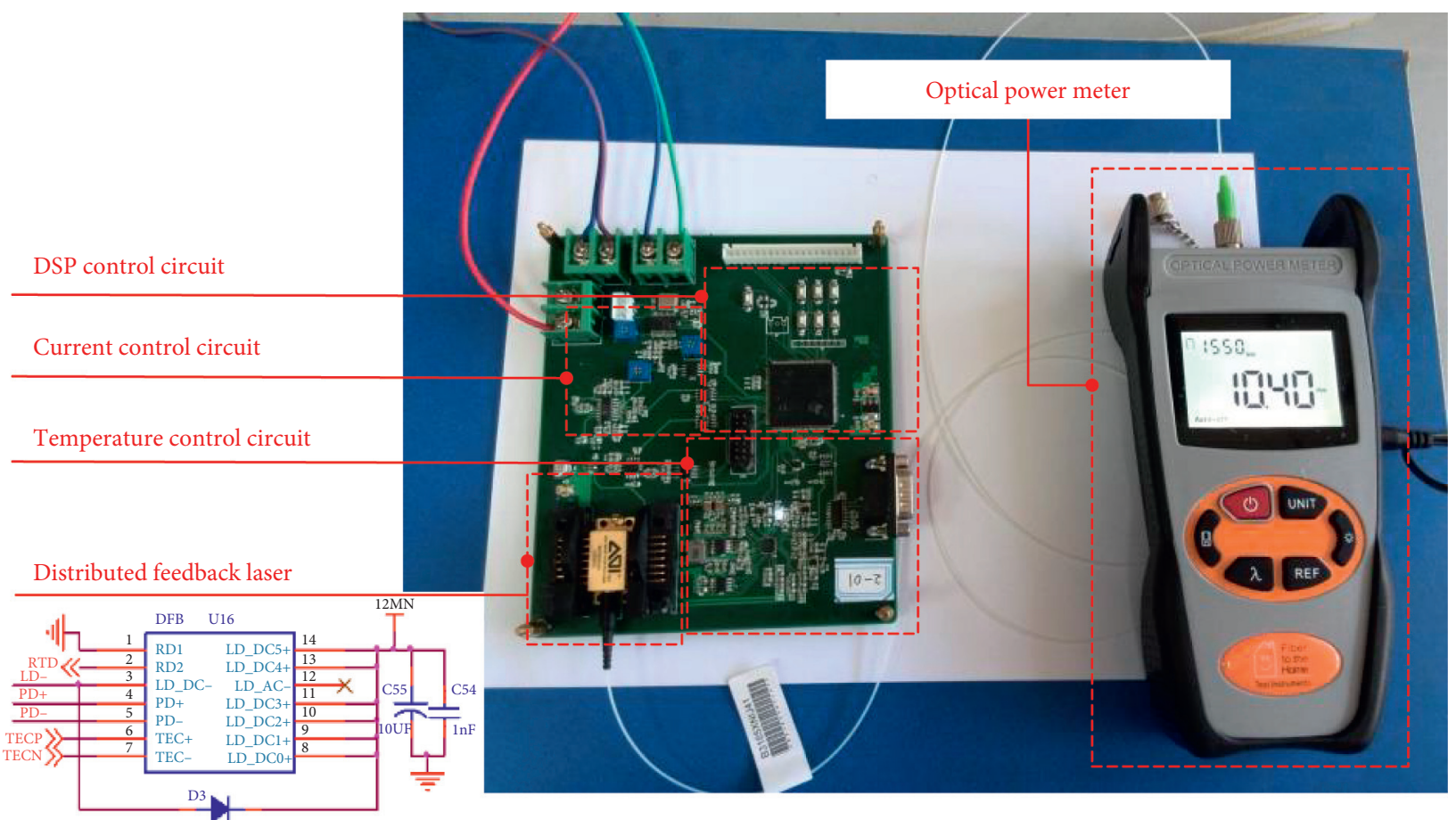

FIgURE 7: Photo of the developed laser driving circuit and optical power test.

laser temperature, where the current is set to be $70 \mathrm{~mA}$. It can also be seen that the optical power of each curve is slightly varied as the centre wavelength is shifted linearly. The factor between wavelength and temperature is about $0.115 \mathrm{~nm} /{ }^{\circ} \mathrm{C}$. This reveals that laser temperature determines the centre wavelength significantly and determines the optical power slightly. The result as shown in the figure proves good stability and linearity of the temperature controller.
4.3. Spectroscopy Test with Varying Laser Current. Then, the driving current of the laser was changed and the corresponding spectrum was measured as shown in Figure 10; current modulation causes wavelength shifting while the temperature is maintained at $25^{\circ} \mathrm{C}$. The current is increased from $40 \mathrm{~mA}$ to $80 \mathrm{~mA}$ with the step of $10 \mathrm{~mA}$. Meanwhile, the optical intensity is also largely affected by tuning the driving current. While the driving current increases, the optical intensity rises proportionally. The factor between 


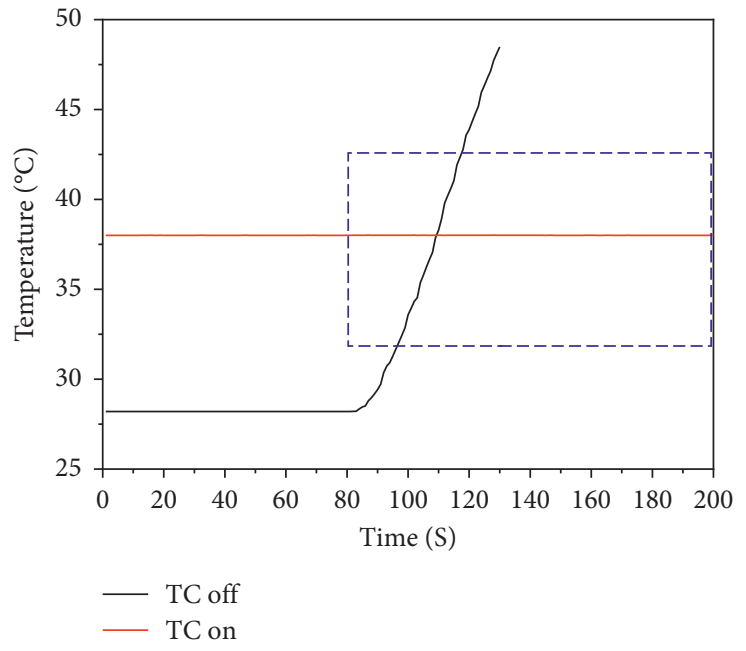

(a)

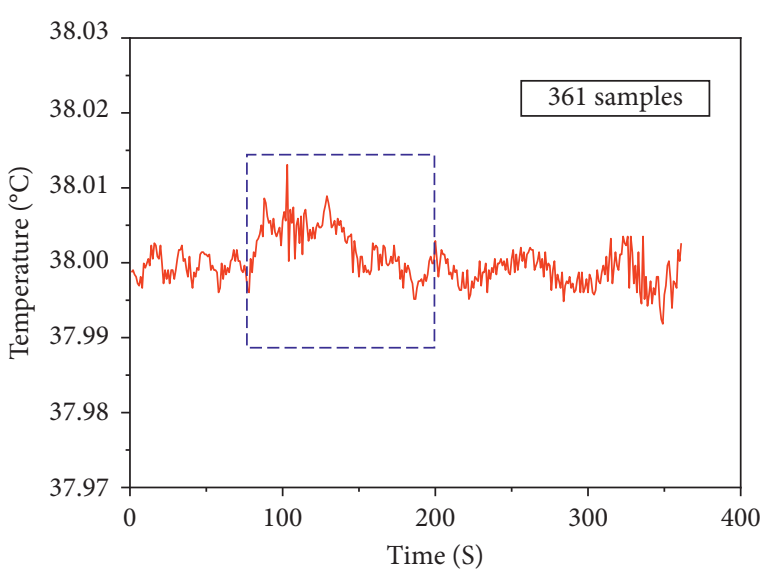

(b)

FIgURE 8: Laser temperature control contrast test (a) and temperature fluctuation under temperature control (b).

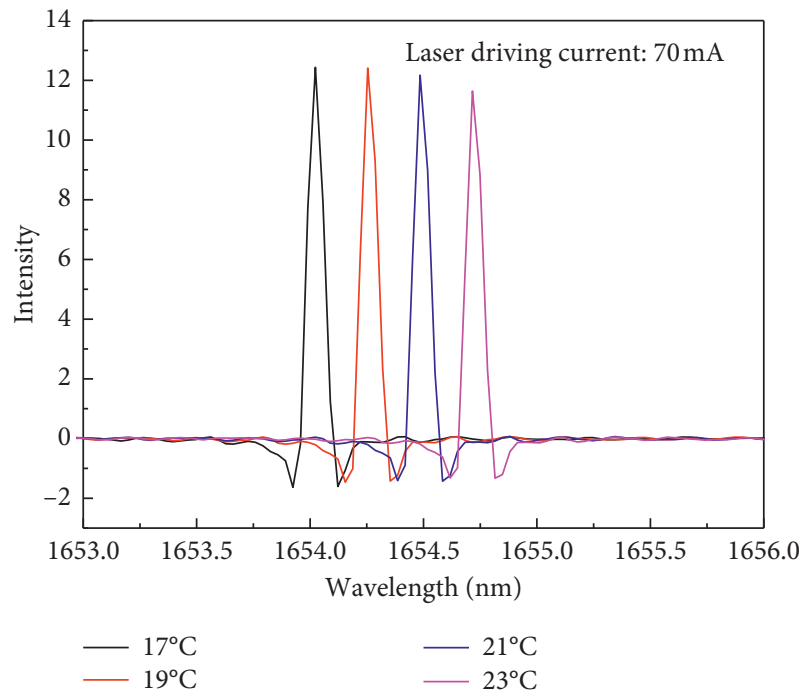

Figure 9: Under the driving current of $70 \mathrm{~mA}$, the measured emitting spectrum under different operation temperatures of $17-23^{\circ} \mathrm{C}$

wavelength and current is about $0.0140 \mathrm{~nm} / \mathrm{mA}\left(T=25^{\circ} \mathrm{C}\right)$. In gas-sensing experiments, the wavelength of the laser is tuned close to the absorption line by adjusting laser temperature to a specific value firstly. Then, the laser temperature is fixed at this point during the experiment. The driving current is periodically changed in order to tune the wavelength. In this way, the wavelength periodically scans across the gas absorption line for the absorption phenomenon. The current is modulated 2000 times per second, which is performed by a DSP timer. The test result proves good efficiency and performance of the developed laser current control circuit.

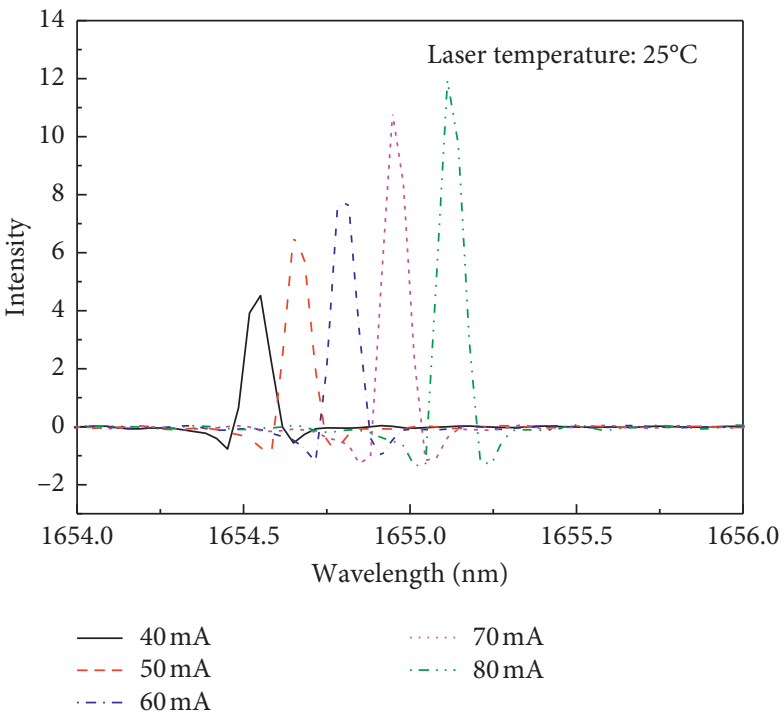

FIgURE 10: Spectrum measurement while the laser driving current increases from $40 \mathrm{~mA}$ to $80 \mathrm{~mA}$.

4.4. Spectroscopy Stability Test. Experiments of spectroscopy stability were carried out to investigate the current driving performance along with the temperature control module. Spectroscopy stability is crucial for TDLAS experiments due to the fact that the shifted centre wavelength of the laser can result inaccuracy gas absorption measurement. The curves of the laser output wavelength versus the injection current is shown in Figure 11. It can be seen that the wavelength under constant temperature and injection current is repeatable. The wavelength was measured five times every 10 minutes, and the curves indicate the good stability and replication of the DFB driver. 


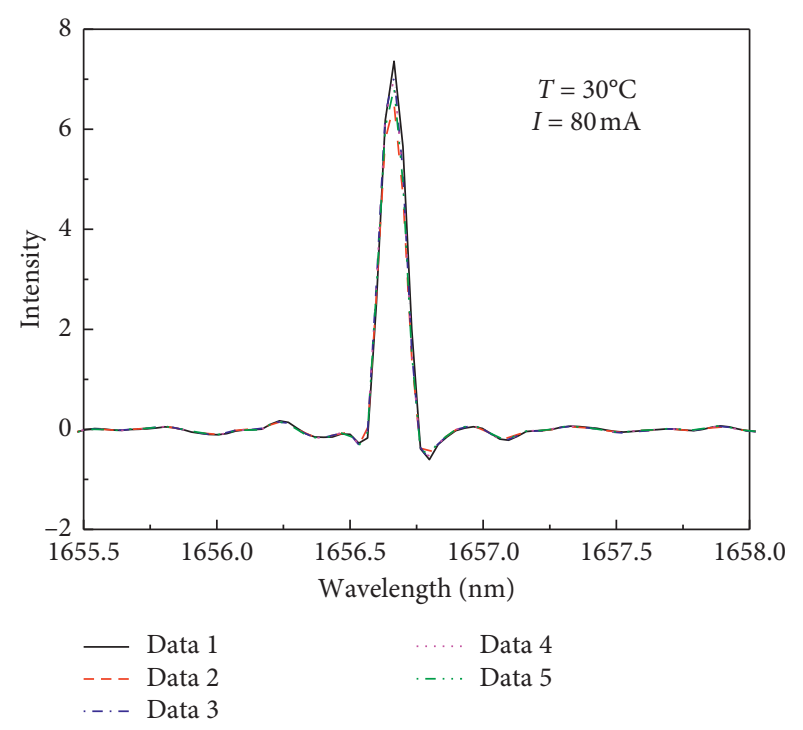

FIGURE 11: Spectroscopy stability test of the DFB laser under constant laser and temperature by using the developed circuit.

\section{Conclusions}

Based on the TDLAS technique, laser current control and temperature control circuits have been developed to control the output wavelength of the laser diode. The wavelength of the laser diode is at $1654 \mathrm{~nm}$, which is applied for methane detection. Laser current and temperature control circuits have been developed and tested accordingly. In addition, a linear power supply circuit has been developed to ensure the stability of the system. Spectroscopy measurements have been performed by changing the laser's current and temperature. Experiments show that the centre wavelength of the laser can be effectively tuned by using the self-developed circuit instead of the commercial instruments. The laser's output wavelength can be controlled by temperature at $0.115 \mathrm{~nm} /{ }^{\circ} \mathrm{C}$ and be controlled by current at $0.0140 \mathrm{~nm} / \mathrm{mA}$. The laser's temperature fluctuation can be limited within the range of -0.02 to $0.02^{\circ} \mathrm{C}$, and good operation stability was observed through 15 hours monitoring on the emitting wavelength of the laser. The laser current can be injected within the range from 40 to $80 \mathrm{~mA}$, and the centre wavelength of the laser was measured accordingly. The proposed circuit can also be applied to drive other DFB lasers for gas detection experiments by changing the parameters and components of the circuit.

\section{Data Availability}

Related data are all included in this article as figures.

\section{Conflicts of Interest}

The authors declare that there are no conflicts of interest regarding the publication of this paper.

\section{Acknowledgments}

The authors express their gratitude to the Ministry of Emergency Management of the People's Republic of China (2012-129), Jilin Jianzhu University (201810191114); the National Key Technology R\&D Program of the Ministry of Science and Technology of China, and the Education Department of Jilin Province of China (JJKH20180573KJ and JJKH20170240KJ) for the generous support of this work.

\section{References}

[1] C. Lou, C. Jing, X. Wang et al., "Near-infrared tunable diode laser absorption spectroscopy-based determination of carbon dioxide in human exhaled breath," Biomedical Optics Express, vol. 10, no. 11, pp. 5486-5496, 2019.

[2] S. Zhou, W. B. Yang, C. X. Liu, L. Zhang, B. L. Yu, and J. S. Li, " $\mathrm{CO}_{2}$-broadening coefficients for the $\mathrm{NO}_{2}$ transitions at $6.2 \mu \mathrm{m}$ measured by mid-infrared absorption spectroscopy," Journal of Quantitative Spectroscopy \& Radiative Transfer, vol. 242, Article ID 106754, 2020.

[3] J. Li, S. Yang, Z. Du et al., "Quantitative analysis of ammonia adsorption in Ag/AgI-coated hollow waveguide by mid-infrared laser absorption spectroscopy," Optics and Lasers in Engineering, vol. 121, pp. 80-86, 2019.

[4] A. M. Julia, F. Prieto, and M. Rueda, "In situ surface enhanced infrared absorption spectroscopy study of the adsorption of cytosine on gold electrodes," Journal of Electroanalytical Chemistry, vol. 849, Article ID 113362, 2019.

[5] Q. Shi, H. M. Long, T. J. Chun, and Z. F. Gao, "Catalytic combustion of chlorobenzene with $\mathrm{VOx} / \mathrm{CeO} 2$ catalysts influence of catalyst synthesis metho," International Journal of Chemical Reactor Engineering, vol. 17, no. 12, Article ID 20190084, 2019.

[6] G. C. Wei, Q. L. Zhang, D. H. Zhang et al., "The influence of annealing temperature on copper-manganese catalyst towards the catalytic combustion of toluene: the mechanism study," Food Hydrocolloid, vol. 497, Article ID 143777, 2020.

[7] G. Yu, J. Wang, J. Liu et al., "Paper-structured catalyst based on $\mathrm{CeO}_{2}-\mathrm{ZrO}_{2}$ fibers for soot combustion," Catalysis Letters, vol. 149, no. 12, pp. 3543-3555, 2019.

[8] S. Afshar, H. A. Zamani, and K. M. Hassan, "Electrochemical determination of sulfapyridine using a new approach of modified electrode based on amplification with room temperature ionic liquid and $\mathrm{ZnO}$ nanoparticle," Analytical and Bioanalytical Chemistry, vol. 11, no. 12, pp. 1781-1790, 2019.

[9] K. K. Kumar, M. Devendiran, R. A. Kalaivani, and S. S. Narayanan, "Enhanced electrochemical sensing of dopamine in the presence of AA and UA using a curcumin functionalized gold nanoparticle modified electrode," New Journal of Chemistry, vol. 43, no. 48, pp. 19003-19013, 2019.

[10] R. Chokkareddy, N. Thondavada, N. K. Bhajanthri, and G. G. Redhi, "An amino functionalized magnetite nanoparticle and ionic liquid based electrochemical sensor for the detection of acetaminophen," Analytical Methods, vol. 11, no. 48, pp. 6204-6212, 2019.

[11] M. Aslinezhad, "High sensitivity refractive index and temperature sensor based on semiconductor metamaterial perfect absorber in the terahertz band," Optics Communications, vol. 463, Article ID 125411, 2020. 
[12] N. Moser, C. L. Leong, Y. Hu et al., "Complementary metaloxide-semiconductor potentiometric field-effect transistor array platform using sensor learning for multi-ion imaging," Analytical Chemistry, vol. 92, no. 7, pp. 5276-5285, 2020.

[13] H. M. Jeong, H. C. Kwon, B. R. Xu et al., “Taste sensor based on the floating gate structure of a lateral double-diffused metal-oxide semiconductor," Sensors and Actuators B: Chemical, vol. 308, Article ID 127661, 2020.

[14] A. Upadhyay, M. Lengde, D. Wilson et al., "A new RAM normalized if-WMS technique for the measurement of gas parameters in harsh environments and a comparison with $2 \mathrm{f} /$ 1f," IEEE Photonics Journal, vol. 10, no. 6, Article ID 6804611, 2018.

[15] L. Lan, H. Ghasemifard, Y. Yuan et al., "Assessment of urban $\mathrm{CO}_{2}$ measurement and source attribution in munich based on TDLAS-WMS and trajectory analysis," Atmosphere, vol. 11, no. 1, p. $58,2020$.

[16] W. Zhao, L. Xu, A. Huang et al., "A WMS based TDLAS tomographic system for distribution retrievals of both gas concentration and temperature in dynamic flames," IEEE Sensors Journal, vol. 20, no. 8, pp. 4179-4188, 2020.

[17] M. M. Sentko, S. Schulz, B. Stelzner, C. Anderlohr, M. Vicari, and D. Trimis, "Determination of temperature and waterconcentration in fuel-rich oxy-fuel methane flames applying TDLAS," Combustion and Flame, vol. 214, pp. 336-345, 2020.

[18] F. Zheng, X. B. Qiu, L. G. Shao et al., "Measurement of nitric oxide from cigarette burning using TDLAS based on quantum cascade laser," Optics \& Laser Technology, vol. 124, Article ID 105963, 2020

[19] L. G. Shao, B. Fang, F. Zheng et al., "Simultaneous detection of atmospheric $\mathrm{CO}$ and $\mathrm{CH}_{4}$ based on TDLAS using a single 2.3 um DFB laser," Spectrochim Acta A, vol. 222, Article ID $117118,2019$.

[20] B. Li, C. Zheng, H. Liu et al., "Development and measurement of a near-infrared $\mathrm{CH}_{4}$ detection system using $1.654 \mu \mathrm{m}$ wavelength-modulated diode laser and open reflective gas sensing probe," Sensors and Actuators B: Chemical, vol. 225, pp. 188-198, 2016.

[21] T. Numai, "High resonance frequency in a coupled cavity DFB-LD with two phase-shifts," Optical and Quantum Electronics, vol. 52, no. 3, p. 150, 2020.

[22] J. H. Kang, H. Wenzel, E. Freier et al., "Continuous-wave operation of DFB laser diodes based on GaN using $10 \$\{\mid \mathrm{rm}$ th\}\$th-order laterally coupled surface gratings," Optics Letters, vol. 45, no. 4, pp. 935-938, 2020.

[23] M. Chen, S. P. Liu, Y. C. Shi et al., "Study on DFB semiconductor laser based on sampled moire grating integrated with grating reflector," IEEE Journal of Quantum Electronics, vol. 56, no. 1, Article ID 2200109, 2020.

[24] Z. Lintao Zhang and J. C. Cartledge, "Fast wavelength switching of three-section DBR lasers," IEEE Journal of Quantum Electronics, vol. 31, no. 1, pp. 75-81, 1995.

[25] H. Ishii, K. Kasaya, and H. Oohashi, "Narrow spectral linewidth operation," Electronics Letters, vol. 46, no. 10, pp. 714-715, 2010.

[26] Z. Y. Lu, Y. S. Zhang, J. Zeng et al., "High precision dual frequency Doppler lidar based on monolithic integrated twosection DFB lasers," IEEE Photonics Journal, vol. 11, no. 6, 2019.

[27] J. X. Mei, L. Wang, T. Tan, K. Liu, G. S. Wang, and X. M. Gao, "A new method of DFB laser frequency stabilization based on the characteristics of the second harmonic," Spectroscopy and Spectral Analysis, vol. 39, no. 10, pp. 2989-2992, 2019.
[28] B. Jens, C. A. Markus, and J. B. Daniel, Tunable Laser Diodes and Related Optical Sources, pp. 4-151, John Wiley \& Sons, Hoboken, NJ, USA, 2005.

[29] R. Soref and J. Larenzo, "All-silicon active and passive guidedwave components for $\lambda=1.3$ and $1.6 \mu \mathrm{m}$," IEEE Journal of Quantum Electronics, vol. 22, no. 6, pp. 873-879, 1986.

[30] H. Yamamoto, M. Asada, and Y. Suematsu, "Electric-fieldinduced refractive index variation in quantum-well structure," Electronics Letters, vol. 21, no. 13, pp. 579-580, 1985.

[31] K. Chinen, K. Gen-ei, H. Suhara et al., "Low-threshold 1.55$\mu \mathrm{m}$ InGaAsP/InP buried heterostructure distributed feedback lasers," Applied Physics Letters, vol. 51, no. 4, pp. 273-275, 1987. 